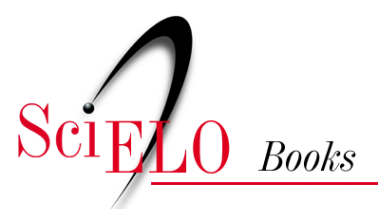

Parte 4 - Pesquisa qualitativa: aplicações em outros estudos geográficos

\title{
Complementaridade das técnicas quantitativas e qualitativas nos estudos ambientais: o solo e o manejo como foco de pesquisa
}

\author{
Thalita Mendes Resende \\ Vania Rosolen
}

\section{SciELO Books / SciELO Livros / SciELO Libros}

RESENDE, T.M., and ROSOLEN, V. Complementaridade das técnicas quantitativas e qualitativas nos estudos ambientais: o solo e o manejo como foco de pesquisa. In: MARAFON, G.J., RAMIRES, J.C.L., RIBEIRO, M.A., and PESSÔA, V.L.S., comps. Pesquisa qualitativa em geografia: reflexões teóricoconceituais e aplicadas [online]. Rio de Janeiro: EDUERJ, 2013, pp. 473-484. ISBN 978-85-7511-443-8. https://doi.org/10.7476/9788575114438.0027.

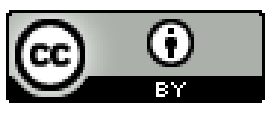

All the contents of this work, except where otherwise noted, is licensed under a Creative Commons Attribution 4.0 International license.

Todo o conteúdo deste trabalho, exceto quando houver ressalva, é publicado sob a licença Creative Commons Atribição $\underline{4.0}$.

Todo el contenido de esta obra, excepto donde se indique lo contrario, está bajo licencia de la licencia $\underline{\text { Creative }}$ Commons Reconocimento 4.0. 


\section{Complementaridade das técnicas quantitativas e qualitativas nos estudos ambientais: o solo e o manejo como foco de pesquisa}

Thalita Mendes Resende

Vania Rosolen

O desenvolvimento de pesquisas em meio ambiente exige conceitos, métodos e aplicação de técnicas inter e multidisciplinares. O paradigma ambiental que ocupa o centro das discussões econômicas, políticas e sociais em todas as esferas de governo é um dos enfoques da geografia. Contudo, ao ampliar o foco sobre os objetos de estudo classificados como ambientais, inúmeras possibilidades existem dentro da geografia, permitindo a aplicação de métodos e técnicas por vezes mais quantitativos ou qualitativos em razão do caráter físico e humano que a caracteriza.

Considerando-se algumas questôes de ordem ambiental de interesse internacional atreladas mais particularmente aos meios tropicais (entendidos como a faixa de terra cuja extensão geográfica situa-se entre as latitudes de $30^{\circ} \mathrm{N}$ e $30^{\circ} \mathrm{S}$ ), destaca-se a conservação do solo. Segundo Lal (1999), os solos, como recurso nos trópicos, são heterogêneos e apresentam diferenças consideráveis em relação à qualidade e às limitaçôes impostas para efetivar suas capacidades produtivas. Em função de características climáticas severas, cultivos com baixa tecnologia e elevada pressão demográfica, são esperados problemas de degradação dos solos e degradação ambiental que resultam em perdas relacionadas ao próprio recurso, como erosão, diminuição do teor de matéria orgânica, 
perda estrutural, perda e desequilíbrio de nutrientes e redução da biodiversidade, assim como reflexos na produtividade, na segurança alimentar e na qualidade ambiental.

A equalização entre os ganhos e as perdas ambientais e sociais relacionadas ao solo depende essencialmente da capacidade de manter sua resiliência em razão de seu uso e dos sistemas de manejo. $\mathrm{O}$ uso do conceito de resiliência nos estudos ambientais e, por conseguinte, para a geografia é adequado por tratar da esfera social e ambiental, ou seja, é um sistema interdependente entre pessoas e natureza, verdadeiro para comunidades locais e seus ecossistemas de entorno, mas válido também em escala global relacionando a aceleração das atividades humanas sobre a Terra (Folke et al., 2010). Em vista dessa relação, é irracional separar o ecológico e o social ou tentar explicá-los independentemente, mesmo que para fins analíticos.

A agricultura (cultivos e pastagens) ocupa hoje 38\% da superfície da Terra, configurando-se como o uso da terra de maior extensão em área (Fao, 2011). Ocupa as melhores terras disponíveis, pois o restante delas possui grandes limitações por se tratar de desertos, montanhas, tundras, cidades, reservas ambientais ou, ainda, outras impróprias para a agricultura. Apesar desse uso intensivo do solo, até recentemente, a comunidade científica não podia medir, monitorar e analisar as complexas ligações entre os sistemas agrícola e alimentar na escala global, enquanto hoje existem dados que permitem avaliar o padrão e as tendências da agricultura e do ambiente (Foley et al., 2011).

Os impactos ambientais relacionados à agricultura podem ser de duas formas (West et al., 2010; Foley et al., 2011): (1) causados pela expansão, quando áreas com vegetação original são convertidas em áreas agrícolas ou; (2) causados pela intensificação, quando o aumento da produção é resultado do aumento do uso de fertilizantes, pesticidas, irrigação e mecanização. No primeiro caso, a substituição de áreas virgens por terras agrícolas causa severos impactos na biodiversidade e nos estoques de Carbono orgânico do solo. A agricultura, no mundo, converteu cerca de $70 \%$ das áreas originalmente cobertas por gramíneas, 50\% das savanas, $45 \%$ das florestas decíduas temperadas e $27 \%$ da floresta tropical, e acredita-se que, nos trópicos, cerca de $80 \%$ das novas áreas agrícolas substituirão áreas originais que concentram elevada biodiversidade de serviços ecossistêmicos (Ramankutty et al., 2008). No Brasil, admite-se que $75 \%$ da emissão de $\mathrm{CO}_{2}$ venham da agricultura e da mudança do uso da terra, o que significa que a agricultura representa mais de dois terços do total das emissóes nacionais (Cerri et al., 2007). A intensificação agrícola, aumentada drasticamente nas últimas décadas, está atrelada ao crescimento do uso de energia, à pressão sobre os recursos hídricos e à poluição (Matson et al., 1997).

Em virtude do aumento de área e de tecnologias, a produção agrícola em todo o mundo vem crescendo substancialmente desde 1960, embora não sem contradições, 
uma vez que uma em sete pessoas é malnutrida ou faminta (Foley et al., 2011) e estas concentram-se, em grande número, nas regiôes tropicais.

A expansão e a intensificação agrícolas são duas características importantes da agricultura praticada no cerrado. As formações savânicas da América do Sul são apresentadas como uma das maiores áreas de potencial de desenvolvimento da agricultura e consideradas alternativas para evitar o aumento do desmatamento das florestas tropicais úmidas com elevada biodiversidade (López-Hernández, 1998).

O cerrado, considerado inapto para a agricultura até aproximadamente a década de 1960, a partir de então, torna-se área de utilização intensiva agrícola conduzida pelo desenvolvimento dos segmentos industriais de máquinas agrícolas, de agroquímicos e de melhorias fitossanitárias fortemente apoiados pelas políticas governamentais e grandes grupos ou empresas nacionais e estrangeiros que comandam o agronegócio (Brossard e López-Hernández, 2005; Pereira, 2012). Imensas áreas de vegetação original foram e continuam sendo desmatadas para dar espaço a monoculturas de grãos (soja e milho, principalmente), algodão, pastagens melhoradas associadas com pastagens degradadas, implantação de florestas homogêneas para produção de carvão e celulose e, nos últimos anos, a expansão da cana-de-açúcar. É inquestionável o desenvolvimento econômico relacionado ao agronegócio regional, mas é inegável também que tenha havido uma série de impactos ambientais relacionados especificamente ao solo.

Interpretar os impactos ambientais relacionados ao solo é relacionar parâmetros indicadores de qualidade do solo com algumas características inerentes ao próprio solo que determinam maior ou menor susceptibilidade e, enfim, avaliar o uso da terra e as condiçôes históricas que levaram à conversão e às opçôes de manejo escolhidas pelo produtor. É nesse sentido que o desenvolvimento de pesquisas sobre conservação dos solos e avaliação da qualidade ambiental deve se pautar pelo uso de técnicas quantitativas, que fornecem valores de referência para a classificação do estado dos ambientes, e técnicas qualitativas, que fornecem informações sobre as ações que podem desencadear o impacto e as motivações sociais, políticas e econômicas associadas a elas.

\section{Técnicas quantitativas e qualitativas tendo o solo como objeto da análise ambiental}

\section{Técnicas quantitativas}

Avaliar a qualidade é dar um caráter de utilidade do solo para o desenvolvimento das atividades humanas. Assim, o solo perde a qualidade quando há o declínio da capacidade produtiva causada pelo mau uso. A avaliação da qualidade do solo é feita por meio de parâmetros de qualidade, que podem ser químicos, físicos e biológicos, e 
o monitoramento do comportamento desses parâmetros ao longo do tempo permite avaliar a conservação do solo. $\mathrm{O}$ uso adequado garante a resiliência desse recurso e é condição para manter a capacidade de produção de alimentos.

Os indicadores de qualidade podem ser quantitativos e objetivos ou qualitativos e subjetivos. O termo indicador é originário do latim indicare, que significa descobrir, apontar, anunciar, estimar. Os indicadores podem comunicar ou informar sobre o progresso em direção a determinada meta (Hammond et al., 1995). Nessa etapa do estudo, optou-se por indicadores quantitativos.

Diante da necessidade de avaliar os impactos relacionados ao uso do solo agrícola no cerrado visando atribuir um caráter mais amplo ao impacto ambiental e manter a capacidade produtiva, optou-se pelo solo como objeto de análise. Dentre os parâmetros possíveis de ser avaliar, escolheu-se como indicador de qualidade o Carbono orgânico do solo, porque este, em ambientes tropicais, é um componente fundamental do potencial produtivo dos solos. A matéria orgânica do solo, composta por aproximadamente $90 \%$ de Carbono, tem grande importância como fonte de nutrientes para vegetais, complexação de elementos tóxicos e micronutrientes, estabilidade estrutural, infiltração e retenção de água, aeração e serve como fonte de energia aos organismos heterotróficos. Sob vegetação natural, o conteúdo de matéria orgânica do solo encontra-se estável, em um balanço que envolve adição e perda. A conversão de ecossistemas naturais em sistemas agrícolas altera esse balanço e ocasiona uma redução acentuada quando se utilizam métodos de preparo com intenso revolvimento do solo e sistemas de cultura com baixa adição de resíduos vegetais. As quantidades de Carbono adicionadas ao solo e perdidas se dão em função da umidade, da temperatura e das práticas de manejo. Em regióes tropicais, a perda do Carbono da matéria orgânica é elevada, e esse comportamento justifica sua escolha como parâmetro de qualidade nos solos do cerrado.

A aplicação do indicador Carbono do Solo para avaliar a qualidade baseia-se no teor total (Carbono Orgânico Total - COT \%) e no Carbono Isotópico $\left({ }^{13} \mathrm{C}\right)$, que fornece informações sobre a fonte do Carbono. Usando-se o C\%, pode-se avaliar o quanto de Carbono foi incorporado ou perdido na conversão e o ${ }^{13} \mathrm{C}$ permite avaliar se houve a incorporação de Carbono de outra fonte (agrícola, nesse caso) que não seja aquela da vegetação original (nesse caso, cerradão). Ambos são determinados por espectrômetro de massa.

Obtém-se o teor total do Carbono Orgânico estimando-se a diferença entre o Carbono Total e o Carbono Inorgânico. Expressa-se o resultado em porcentagem (\%) de Carbono Orgânico (C).

Quanto à determinação da fonte do Carbono, parte-se do conhecimento que na natureza existem dois isótopos estáveis de Carbono: ${ }^{12} \mathrm{C}$, que é o mais leve e apresenta 
a maior proporção no $\mathrm{C}$ total existente na natureza $(98,89 \%)$; e o ${ }^{13} \mathrm{C}$, que representa apenas $1,11 \%$. Essas proporçōes se mantêm relativamente estáveis em qualquer resíduo orgânico. Para avaliar essa modificação na proporção de ${ }^{13} \mathrm{C} /{ }^{12} \mathrm{C}$ em uma amostra, adota-se como referência um padrão internacional. Esse padrão é uma rocha calcária dolomítica (Belemnita) encontrada em uma formação geológica denominada Pee Dee Belemnite (PDB), na Carolina do Norte (EUA), que tem uma relação molar de ${ }^{13} \mathrm{C} /{ }^{12} \mathrm{C}$ (R) constante de 0,01124 . Os desvios da proporção de ${ }^{13} \mathrm{C} /{ }^{12} \mathrm{C}$ em relação ao padrão são conhecidos como unidades $\delta$ (letra grega delta minúscula), e, como essas diferenças são valores muito pequenos, convencionou-se expressá-los em partes por mil (\%o) (equação 1). As relações molares ${ }^{13} \mathrm{C} /{ }^{12} \mathrm{C}$ do $\mathrm{CO}_{2}$ atmosférico e das plantas são inferiores ao padrão $\mathrm{PDB}$, e, por isso, seus valores de $\delta^{13} \mathrm{C}$ são negativos. No caso do $\mathrm{CO}_{2}$ atmosférico, os valores observados são próximos de $-7 \%$ \% $\mathrm{e}$, no das plantas, variam de -11 a $-35 \%$ o $\delta$ (Farquhar, Ehleringer e Hubick, 1989).

Equação 1: $\quad \delta \%$ o $=\left(\frac{\text { R amostra }}{\mathrm{R} \text { padrão }}-1\right) \times 1.000$

onde $\mathrm{R}$ é a relação isotópica ${ }^{13} \mathrm{C} /{ }^{12} \mathrm{C}$.

Pôde-se aplicar a técnica de abundância natural de ${ }^{13} \mathrm{C}$ nos estudos da dinâmica da matéria orgânica do solo por causa da diferença na relação dos isótopos de $\mathrm{C}$ que existem nos diversos grupos de plantas de ciclos fotossintéticos $\mathrm{C}_{3}$ e $\mathrm{C}_{4}$. Plantas de ciclo $\mathrm{C}_{3}$ apresentam variações na abundância isotópica de ${ }^{13} \mathrm{C}$ de -20 a $-34 \delta$ (média de -27 $\delta)$. Grande parte das espécies florestais pertence a esse grupo. As plantas de ciclo $\mathrm{C}_{4}$ discriminam menos $0{ }^{13} \mathrm{C}$ e apresentam valores que variam de $-9 \mathrm{a}-17 \delta$ (média de -13 ठ) (Smith e Epstein, 1971).

Devem-se fazer as coletas das amostras de solo: (1) em uma área considerada valor de referência; e (2) nos diferentes usos do solo que se queira avaliar, cujos valores serão confrontados com os resultados da referência. Devem-se realizar as coletas na camada superficial do solo até aproximadamente $30 \mathrm{~cm}$ de profundidade, pois os maiores teores de Carbono Orgânico encontram-se estocados na superfície do solo, e o desflorestamento e a perda por mineralização se refletem de forma mais rápida e acentuada nessa camada (Bernoux et al., 2002). Em cada categoria de uso, devem-se coletar pelo menos três amostras para possibilitar o cálculo da média e do desvio padrão.

A aplicação dessas técnicas quantitativas escolhidas como parâmetros de qualidade dos solos em um estudo na Bacia do Ribeirão Bom Jardim, no município de Uberlândia/MG, resultou nos dados apresentados a seguir (tabela 1). 
Sob vegetação original de cerrado arbóreo, na profundidade entre $0-10 \mathrm{~cm}$, determinaram-se os teores de Carbono (C\%) de 1,5 a 2\% (média de 1,75\%; desvio padrão de 0,26); e, na profundidade entre 20-30 cm, de 0,77 a 0,92\% (média de 0,86\%; desvio padrão de 0,08$)$. No solo recoberto com pastagem melhorada, entre $0-10 \mathrm{~cm}$, C\% variou entre 0,91 e $1,43 \%$ (média de $1,13 \%$; desvio padrão de 0,27 ); e, entre 20 $30 \mathrm{~cm}$, de 0,67 a $0,75 \%$ (média de $0,7 \%$; desvio padrão de 0,04 ). A perda média aproximada de Carbono nas camadas de solo de pastagem melhorada em relação às camadas do solo do cerrado foi de aproximadamente $22 \%$ nos primeiros $10 \mathrm{~cm}$ de profundidade e de $19 \%$ na profundidade entre $20-30 \mathrm{~cm}$.

No solo com pastagem degradada, houve nitidamente um empobrecimento de C, tanto em superfície quanto em subsuperfície (63,43\% e 50\%, respectivamente). $\mathrm{Na}$ profundidade entre $0-10 \mathrm{~cm}$, os teores de $\mathrm{C}$ variaram de 0,61 a $0,66 \%$ (média de $0,64 \%$; desvio padrão de 0,03 ); e, entre $20-30 \mathrm{~cm}$, de 0,41 a $0,45 \%$ (média de $0,43 \%$; desvio padrão de 0,02 ). No solo cultivado com soja, os teores de $\mathrm{C}$ na superfície, entre 0-10 cm, variaram de 2,06 a 2,36\% (média de 2,23\%; desvio padrão de 0,15); e, entre 20-30 cm, de 1,54 a 1,74\% (média de 1,64\%; desvio padrão de 0,10).

Tabela 1 - Análise de teor e origem do Carbono sob diferentes usos da terra e cobertura vegetal - Bacia do Ribeirão Bom Jardim

\begin{tabular}{|c|c|c|c|c|}
\hline \multirow[b]{2}{*}{ Ponto } & \multirow{2}{*}{$\begin{array}{l}\text { Identificação } \\
\text { (uso atual) }\end{array}$} & \multirow{2}{*}{$\begin{array}{l}\text { Prof.*** } \\
(\mathrm{cm})\end{array}$} & \multicolumn{2}{|c|}{ Teor e origem do Carbono } \\
\hline & & & $\begin{array}{l}\text { C Total } \\
(\mathrm{g} / 100 \mathrm{~g})\end{array}$ & $\begin{array}{l}\delta^{13} \mathrm{C} \\
(\% o)\end{array}$ \\
\hline C-1-1 & Cerrado & $0-10$ & 1,74 & $-26,08$ \\
\hline C-1-2 & Cerrado & $20-30$ & 0,92 & $-25,04$ \\
\hline C-2-1 & Cerrado & $0-10$ & 2,01 & $-26,18$ \\
\hline C-2-2 & Cerrado & $20-30$ & 0,90 & $-24,84$ \\
\hline C-3-1 & Cerrado & $0-10$ & 1,50 & $-26,33$ \\
\hline C-3-2 & Cerrado & $20-30$ & 0,77 & $-24,44$ \\
\hline Média & \multirow{2}{*}{ Cerrado } & \multirow{2}{*}{$0-10$} & 1,75 & $-26,20$ \\
\hline $\mathrm{DP}^{*}$ & & & 0,26 & 0,13 \\
\hline Média & \multirow{2}{*}{ Cerrado } & \multirow{2}{*}{$20-30$} & 0,86 & $-24,77$ \\
\hline $\mathrm{DP}^{*}$ & & & 0,08 & 0,31 \\
\hline$S-1-1$ & Soja/milho** & $0-10$ & 2,28 & $-20,54$ \\
\hline$S-1-2$ & Soja/milho** & $20-30$ & 1,74 & $-18,48$ \\
\hline$S-2-1$ & Soja/milho** & $0-10$ & 2,36 & $-16,66$ \\
\hline$S-2-2$ & Soja/milho** & $20-30$ & 1,54 & $-14,81$ \\
\hline$S-3-1$ & Soja/milho** & $0-10$ & 2,06 & $-17,43$ \\
\hline$S-3-2$ & Soja/milho** & $20-30$ & 1,65 & $-16,13$ \\
\hline
\end{tabular}




\begin{tabular}{|c|c|c|c|c|}
\hline Média & \multirow{2}{*}{ Soja/milho** } & \multirow{2}{*}{$0-10$} & 2,23 & $-18,21$ \\
\hline $\mathrm{DP}^{*}$ & & & 0,15 & 2,06 \\
\hline Média & \multirow{2}{*}{ Soja/milho** } & \multirow{2}{*}{$20-30$} & 1,64 & $-16,47$ \\
\hline \multirow[t]{2}{*}{ DP* } & & & 0,10 & 1,86 \\
\hline & Pastagem manejada & $0-10$ & 0,91 & $-13,34$ \\
\hline PM-1-2 & Pastagem manejada & $20-30$ & 0,69 & $-13,05$ \\
\hline PM-2-1 & Pastagem manejada & $0-10$ & 1,06 & $-13,36$ \\
\hline PM-2-2 & Pastagem manejada & $20-30$ & 0,67 & $-13,17$ \\
\hline PM-3-1 & Pastagem manejada & $0-10$ & 1,43 & $-13,39$ \\
\hline PM-3-2 & Pastagem manejada & $20-30$ & 0,75 & $-12,85$ \\
\hline Média & \multirow{2}{*}{ Pastagem manejada } & \multirow{2}{*}{$0-10$} & 1,13 & $-13,37$ \\
\hline $\mathrm{DP}^{*}$ & & & 0,27 & 0,03 \\
\hline Média & \multirow{2}{*}{ Pastagem manejada } & \multirow{2}{*}{$20-30$} & 0,70 & $-13,03$ \\
\hline \multirow[t]{2}{*}{$\mathrm{DP}^{*}$} & & & 0,04 & 0,16 \\
\hline & Pastagem degradada & $0-10$ & 0,63 & $-13,93$ \\
\hline PD-1-2 & Pastagem degradada & $20-30$ & 0,45 & $-14,62$ \\
\hline PD-2-1 & Pastagem degradada & $0-10$ & 0,61 & $-13,96$ \\
\hline PD-2-2 & Pastagem degradada & $20-30$ & 0,41 & $-14,38$ \\
\hline PD-3-1 & Pastagem degradada & $0-10$ & 0,66 & $-14,57$ \\
\hline PD-3-2 & Pastagem degradada & $20-30$ & 0,44 & $-13,50$ \\
\hline Média & \multirow{2}{*}{ Pastagem degradada } & \multirow{2}{*}{$0-10$} & 0,64 & $-14,15$ \\
\hline $\mathrm{DP}^{*}$ & & & 0,03 & 0,36 \\
\hline Média & \multirow{2}{*}{ Pastagem degradada } & \multirow{2}{*}{$20-30$} & 0,43 & $-14,17$ \\
\hline $\mathrm{DP}^{*}$ & & & 0,02 & 0,59 \\
\hline
\end{tabular}

Legenda: ${ }^{*}$ desvio padrão; ${ }^{* *}$ soja rotação com milho; ${ }^{* * *}$ profundidade.

Em relação à origem do Carbono, o enriquecimento ou o empobrecimento no solo estão acompanhados de mudanças da assinatura isotópica $\left(\delta^{13} \mathrm{C}\right) . \mathrm{Na}$ área de estudo, a matéria orgânica do solo (MOS) da área recoberta por cerrado apresentou $\delta^{13} \mathrm{C}$ entre -26,08 e -26,33\%o na profundidade entre 0-10 cm (média de -26,20\%o; desvio padrão de 0,13); e, entre -24,44 e -25,04\%o, entre 20-30 cm (média de -24,77\%o; desvio padrão de 0,31$). \mathrm{O} \delta^{13} \mathrm{C}$ da matéria orgânica dos solos atualmente usados com pastagem melhorada, pastagem degradada e soja e milho difere daqueles determinados sob cerrado e indica clara tendência de alteração da decomposição e incorporação da MOS nas áreas convertidas. Houve mudança nítida de incorporação de plantas $\mathrm{C}_{4}$ originadas da gramínea forrageira Brachiaria spp. no solo convertido em pastagem melhorada (15 anos de conversão), que apresentou $\delta^{13} \mathrm{C}$ entre -12,85 e -13,39\%o (média de $-13,37 \%$ entre $0-10 \mathrm{~cm}$ e de $-13,03 \%$ entre $20-30 \mathrm{~cm}$ de profundidade). Determinou- 
-se assinatura isotópica similar no solo com pastagem degradada (40 anos de conversão), que apresentou $\delta^{13} \mathrm{C}$ entre $-13,50$ e -14,62\%o (média de -14,15\%o entre $0-10 \mathrm{~cm}$ e de $14,17 \%$ o entre $20-30 \mathrm{~cm}$ de profundidade). $\mathrm{Na}$ área cultivada com soja e milho (10 anos de conversão com sistema de plantio direto), determinou-se assinatura isotópica, que indica mistura de vegetação resultante da incorporação no solo de raízes e resíduos de soja $\left(\mathrm{C}_{3}\right)$ e milho $\left(\mathrm{C}_{4}\right)$. Os valores mínimos e máximos que se obtiveram nas profundidades entre 0-10 e 20-30 cm foram, respectivamente, -14,81 e -20,54\%o (a média que se obteve nas profundidades entre $0-10 \mathrm{~cm}$ foi de $-18,21 \%$ e entre $20-30 \mathrm{~cm} \mathrm{de}$ $-16,47 \%$ o). Os resultados indicaram que, após 15 e 40 anos de conversão do cerrado arbóreo em pastagens, houve modificações relacionadas ao decréscimo do teor e à substituição do $\mathrm{C}$ derivado da MOS de espécies arbóreas $\mathrm{C}_{3}$ pelo $\mathrm{C}$ da MOS derivada de gramíneas $\mathrm{C}_{4}$ cultivadas ou não. A conversão em área agrícola não foi acompanhada por diminuição da taxa de Carbono, mas, sim, da qualidade da MOS.

Portanto, as técnicas quantitativas puderam fornecer valores de referência para a classificação do estado de degradação e/ou conservação dos ambientes. Essas técnicas visam garantir a precisão dos resultados, evitar distorções de análise e de interpretação e aumentar a margem de segurança das soluções encontradas.

\section{Técnicas qualitativas}

As pesquisas ambientais não utilizam apenas técnicas quantitativas e estatísticas. Antes de se obterem os dados quantitativos, são necessárias várias etapas que envolvem o uso de técnicas sociais, comumente utilizadas na pesquisa qualitativa. Elas são precursoras para a coleta de informações, a obtenção de resultados e a interpretação dos dados quantitativos. Essas etapas iniciais se inserem num método de pesquisa social que pode utilizar uma infinidade de técnicas para atingir os objetivos propostos.

Para obter uma compreensão global do objeto de estudo, a pesquisa ambiental deve partir de uma metodologia de trabalho baseada no entendimento das características e do arranjo sistêmico da área de interesse e de seu entorno, bem como do subsistema socioeconômico a ela associado (Seabra, 2001).

Portanto, somado às técnicas quantitativas, essenciais na descrição, mensuração e interpretação das variáveis ambientais, faz-se imperativo o uso de técnicas qualitativas, as quais fornecerão informaçôes sobre as ações que podem desencadear o impacto e as motivações sociais, políticas e econômicas a elas associadas.

É necessário descobrir e classificar a relação entre variáveis e, ainda, investigar a relação de causalidade entre fenômenos. Para isso, inúmeros métodos e técnicas qualitativos são direta ou indiretamente utilizados no processo de coleta de dados na pesquisa ambiental. Dentre eles, pretende-se abordar os que são mais utilizados nessa área de 
pesquisa e, muitas vezes, não são sequer descritos como parte da metodologia: observação simples, trabalho de campo e entrevista.

$\mathrm{Na}$ área pesquisada, a aplicação da técnica de observação simples consistiu no reconhecimento da área de pesquisa. Possibilitou a obtenção dos elementos para a definição do problema da pesquisa; favoreceu a construção de hipóteses acerca deste; e direcionou a escolha dos pontos de coleta e a posterior obtenção de dados. Por se tratar de uma técnica pouco sistemática (Gil, 1999), conjugou-se a outras técnicas, quantitativas, para obtenção de dados com maior confiabilidade. A pesquisa científica caminha para um único objetivo, que é a demonstração da verdade por meio da observação e da experimentação (Seabra, 2001).

A observação é o uso dos sentidos com vistas a adquirir os conhecimentos necessários para o cotidiano. Pode, porém, ser utilizada como procedimento científico, na medida em que serve a um objetivo formulado de pesquisa, é sistematicamente planejada, registrada e ligada a proposições mais gerais e é submetida a verificação e controles de validade e precisão (Selltiz et al., 1967).

A técnica de observação constitui elemento fundamental no processo de pesquisa; é imprescindível desde a formulação do problema e passa pela construção de hipóteses, coleta, análise e interpretação dos dados. Todavia, é mais utilizada na fase de coleta de dados. Segundo Gil (1999), sua principal vantagem em relação a outras técnicas é que os fatos são percebidos diretamente, sem qualquer intermediação, o que reduz a subjetividade. Embora possa ser caracterizada como espontânea, informal, não planificada, coloca-se num plano científico, pois vai além da simples constatação dos fatos. Exige-se um mínimo de controle na obtenção dos dados. Além disso, a coleta de dados por observação é seguida de um processo de análise e interpretação, o que lhe confere a sistematização e o controle requeridos dos procedimentos científicos.

Para que houvesse a observação e o reconhecimento da área de pesquisa, fez-se necessária a realização do trabalho de campo. Essa técnica permitiu a interação do pesquisador com o objeto de estudo. É no campo que o pesquisador percebe e apreende os vários aspectos que envolvem seu estudo, tanto naturais quanto sociais.

Compiani (1991) destaca o campo como cenário de geração, problematização e crítica do conhecimento, onde o conflito entre o real e as ideias ocorre com toda a intensidade. Em geografia, os trabalhos de campo são imprescindíveis, pois permitem ao pesquisador se posicionar perante o saber teórico e a realidade vigente, desmitificando a ciência e construindo um saber mais próximo de seu cotidiano. Além disso, possibilitam a percepção da inter-relação entre os aspectos físicos e humanos (objetos de estudo concomitantes).

O trabalho de campo em geografia requer a definição de espaços de conceituação adequados aos fenômenos que se desejam pesquisar e analisar na realidade. É preciso, 
também, reafirmar a necessidade de superação das dicotomias e ambiguidades características da geografia. $\mathrm{O}$ trabalho de campo é instrumento-chave para a superação dessas ambiguidades, sem priorizar a análise dos chamados fatores naturais nem a dos fatores humanos (ou "antrópicos"). Deve basear-se na totalidade do espaço, sem esquecer os arranjos específicos que tornam cada lugar, cidade, bairro ou região uma articulação particular de fatores físicos e humanos em um mundo fragmentado, porém (cada vez mais) articulado. $\mathrm{O}$ trabalho de campo em geografia deve perseguir, portanto, a ideia de particularidade na totalidade (Serpa, 2006).

A realização do trabalho de campo num estudo pedológico é de suma importância para a coleta das amostras de solo. A escolha dos pontos deve estar relacionada com alguns parâmetros, sendo que a variabilidade dos parâmetros no campo garante a precisão e a qualidade dos resultados.

No momento do trabalho de campo, além da observação simples, utilizou-se a técnica de entrevista com os produtores, para que houvesse o reconhecimento do histórico de uso e ocupação do solo. Por meio da entrevista, puderam-se compreender claramente as opções político-econômicas agrícolas adotadas (convencional ou plantio direto), bem como a viabilidade do manejo visando à conservação.

Pode-se definir entrevista como a técnica em que o investigador se apresenta ao investigado e formula perguntas para obter dados de interesse. A entrevista é, portanto, uma forma de interação social. Possibilita o acesso referente aos mais diversos aspectos da vida social, permite a obtenção de dados em profundidade acerca do comportamento humano, sendo eles suscetíveis de classificação e quantificação (Gil, 1999).

O diagnóstico político, social e econômico compreende a elaboração de um perfil atualizado sobre a área de estudo. Devem-se levar em consideração vários aspectos do processo de ocupação e dos meios de produção e apresentar, a partir de condições sociais determinadas, as principais tendências de uso do espaço, suas formas de produção e os modos de vida a elas associados. São essas relações sociais de produção que se manifestam integralmente no uso e na ocupação do espaço e acarretam consequências de natureza ambiental (Seabra, 2001).

As informações sobre as técnicas empregadas devem ser integradas aos dados quantitativos obtidos por análises laboratoriais e formar um quadro coerente da realidade pesquisada.

O uso e a ocupação do solo da área de estudo deverão ser enfocados tanto sob o ponto de vista da situação atual quanto em relação ao processo histórico de formação econômica e territorial. Nesse sentido, o objetivo principal da entrevista com os produtores consistiu em levantar as informações acerca da expansão espaçotemporal da agricultura e da pecuária. Também se devem ressaltar as ações governamentais na criação de infraestruturas rurais e/ou na facilitação de investimentos na produção. Por 
exemplo, na área de estudo, a pastagem manejada que incorporou mais Carbono no solo em relação à pastagem natural é resultado da aplicação de tecnologia agrícola para melhoria de pastagens com brachiaria efetivada pela Empresa Brasileira de Pesquisa Agropecuária (Embrapa), cujos resultados positivos, em relação à qualidade ambiental ou ao aumento da produção, proporcionaram a obtenção de maiores lucros.

\section{Considerações finais}

A definição de parâmetros ou indicadores para medir a qualidade ambiental em sistemas agrícolas não é tarefa simples e de consenso, pois inúmeros fatores mudam ao longo do tempo, como as evoluções tecnológicas, a introdução de novos sistemas de cultura, os erros associados aos diferentes pontos de amostragem e os problemas de capitalização e de financiamento (Brossard e López-Hernández, 2005). Consciente da complexidade dos sistemas naturais e antrópicos, acredita-se que avaliar a qualidade relacionada ao uso do solo é utilizar indicadores que reflitam a melhoria ou a preservação dos parâmetros físicos, químicos e biológicos obtidos na amostra de referência.

As técnicas quantitativas e qualitativas se complementam nos estudos ambientais. O uso dos teores de Carbono Total e assinatura isotópica do Carbono é particularmente útil para avaliar o impacto gerado pela agricultura nas áreas de cerrado convertidas; porém, uma análise mais precisa e completa ocorre quando se associam técnicas qualitativas de pesquisa. Conhecer o histórico do uso fornecido pelo produtor assegura a interpretação dos resultados numéricos. Obter informações por meio de entrevistas permite a correlação entre as técnicas de manejo do solo aplicadas e o nível de degradação ou conservação deste. Finalmente, a capacidade de investimento, o acesso à informação e as tecnologias agrícolas informadas pelo agricultor respaldam as avaliações sobre alguns motivos que levam os proprietários de terra a optar entre técnicas de manejo mais ou menos conservacionistas.

O modelo de agricultura do cerrado pode se revelar conflitivo frente às necessidades de conservação ambiental e desenvolvimento socioeconômico. A expansão recente da fronteira agrícola nesse bioma deve não apenas assegurar a produção, mas também permitir interrogar se o sistema de produção já efetivado provoca e provocará variações nos quadros bioclimáticos e sociais (Brossard e López-Hernández, 2005).

\section{Referências}

BERNOUX, Martial et al. "Brazil's soil carbon stocks". Soil Science Society American Journal, Madison, 2002, v. 66, pp. 888-96. 
BROSSARD, Michel e LÓPEZ-HERNÁNDEZ, Danilo. "Des indicateurs d'évolution du milieu et des sols pour render durable I'usage des savanes d'Amérique du Sud”. Natures Sciences Sociétés, Paris, 2005, v. 13, pp. 266-78.

CERRI, Carlos E. P. et al. "Tropical agriculture and global warming: impacts and mitigation options". Scientia Agricola, Piracicaba, 2007, v. 64, n. 1, pp. 83-99.

COMPIANI, Maurício. "A relevância das atividades de campo no ensino de geologia na formação de professores de ciências". Cadernos do IG/Unicamp, 1991, v. 1, n. 2, pp. 2-25.

FAO - Food and Agriculture Organization of the United Nations (FAOSTAT). Agricultural production. Disponível em http://faostat.fao.org/site/567/default.aspx\#ancor. Acesso em dez. 2011.

FARQUHAR, Graham D.; EHLERINGER, James. R.; HUBICK, Kerry. T. "Carbon Isotope discrimination and photosynthesis". Annual Review of Plant Physiology and Molecular Biology, Palo Alto, 1989, v. 40, pp. 503-37.

FOLEY, Jonathan A. et al. "Solutions for a cultivated planet”. Nature, Londres, 2011, v. 478, pp. 337-42.

FOLKE, Carl et al. "Resilience thinking: integrating resilience, adaptability and transformability”. Ecology and Society, Tempe, 2010, v. 4, n.15, pp. 1-9.

GIL, Antonio C. Métodos e técnicas de pesquisa social. 5 ed. São Paulo: Atlas, 1999.

HAMMOND, Allen et al. Environmental indicators: a systematic approach to measuring and reporting on environmental policy performance in the context of sustainable development. Washington: WRI, 1995, 53 p.

LAL, Rattan. "Managing tropical soil resource for food security and environmental quality". In FORMOSO, M. L. L. e CERRI, C. C. (orgs.). Workshop on tropical soils. Rio de Janeiro: Academia Brasileira de Ciências, 1999, pp. 31-54.

LÓPEZ-HERNÁNDEZ, Danilo. "Agricultura de bajos insumos en el entorno sabana-bosque como un medio para reducir la presión sobre la selva tropical húmeda". Informe Final CONICIT Proyecto RP VII 2900 89, 1998.

MATSON, Pamela A. et al. "Agricultural intensification and ecosystem properties". Science, Washington, 1997, n. 277, pp. 504-9.

PEREIRA, Mirlei F. V. “Os agentes do agronegócio e o uso do território no Triângulo Mineiro/Alto Paranaíba: da moderna agricultura de grãos à expansão recente da cana-de-açúcar”. Revista do Departamento de Geografia (USP), 2012, v. 23, pp. 83-104.

RAMANKUTTY, Navin et al. "Farming the planet: 1. Geographic distribution of global agricultural lands in the year 2000". Global Biogeochemistry Cycles, 2008, v. 22, GB1003.

SEABRA, Giovanni. F. Pesquisa científica: o método em questão. Brasília: Ed. UnB, 2001, 124 p.

SELLTIZ, Claire et al. Métodos de pesquisa nas relações sociais. São Paulo: Herder/EDUSP, 1967, 688 p.

SERPA, Angelo. "O trabalho de campo em geografia: uma abordagem teórico-metodológica". Boletim Paulista de Geografia, São Paulo, 2006, n. 84, pp. 7-24.

SMITH, Bruce N. e EPSTEIN, Samuel. "Two categories of ${ }^{13} \mathrm{C} /{ }^{12} \mathrm{C}$ ratios for higher plants". Plant Physiology, Rockville, 1971, v. 47, pp. 380-4.

WEST, Paul C. et al. "Trading Carbon for food: global comparison of Carbon stocks vs. crop yields on agricultural land”. PNAS, Washington, 2010, v. 107, n. 46, pp. 19645-8. 